\title{
Nanoscale Imaging of Lithium Ion Distribution During In Situ Operation of a Battery Electrode and Electrolyte
}

\author{
Megan E. Holtz ${ }^{1}$, Yingchao Yu², Deniz Gunceler ${ }^{3}$, Jie Gao ${ }^{2}$, Ravishankar Sundararaman ${ }^{3}$, Kathleen A. \\ Schwarz $^{2}$, Tomás A. Arias ${ }^{3}$, Héctor D. Abruña ${ }^{2}$, and David A. Muller ${ }^{1}$ \\ 1. School of Applied and Engineering Physics, Cornell University, Ithaca NY 14853. \\ 2. Department of Chemistry and Chemical Biology, Cornell University, Ithaca NY 14853. \\ 3. Department of Physics, Cornell University, Ithaca NY 14853.
}

Recently, tremendous effort has been made towards in situ transmission electron microscopy (TEM) studies of material systems.[1] In particular, the study of the lithiation and delithiation of battery materials has attracted much attention,[2] since this mechanism often limits electrochemical performance. Here, we use valence energy-filtered TEM (EFTEM) imaging of an aqueous battery cathode in an electrochemical cell to track ions as they move between electrolyte and cathode material. We can directly observe in real time the chemical information about the charge-state of both the cathode material and the ionic species enrichment and depletion in the electrolyte adjacent to the cathode.

A challenge in the development of battery materials is understanding their fundamental mechanisms of operation and degradation. Because most battery materials are microscopically inhomogeneous on the nanoscale, characterization tools that provide operando and localized information from individual grains and particles are required. Here we discuss a modified electrochemical cell holder (Protochips), which uses patterned chips designed to mimic typical electrochemical cells (Figure 1a). We demonstrate cyclic voltammetry of platinum that reproduces typical ex situ results (Figure 1b), providing a rigorous test case for electrochemistry because the features are sensitive to contaminants at the sub-monolayer level.

We image the nanoscale distribution of lithium ions during electrochemical charging and discharging of an aqueous $\mathrm{LiFePO}_{4}$ battery in a TEM liquid flow cell. TEM detection of lithium through liquid is difficult, because lithium is a weak elastic scatterer and multiple scattering from the liquid overwhelms the inelastic core-loss signal in electron energy-loss spectroscopy (EELS).[3] We use valence EELS to track the ions in both the battery electrolyte and the cathode, using electronic structure fingerprints of the solvated ions identified using an ab initio non-linear response theory. For the cathode, this method is analogous to observing color changes during battery cycling on the micron scale, except EFTEM achieves nanometer resolution. We study the cathode material $\mathrm{LiFePO}_{4}$ in aqueous solution in a monochromated FEI Tecnai F20. When $\mathrm{LiFePO}_{4}$ is delithiated to $\mathrm{FePO}_{4}$, a $5 \mathrm{eV}$ energy transition appears in EELS that is not present in the lithiated state (Figure 2a), allowing observation of the state of lithiation using valence EFTEM. At $0 \mathrm{eV}$, the predominantly elastic signal is dominated by the liquid and shows no diffraction contrast (Figure 2b). However, at $5 \mathrm{eV}$ in the optical gap of the liquid, the EFTEM signal highlights the delithiated particles showing strong chemical transitions (Figure 2c).

Equipped with the electrochemical cell holder, nanoscale spectroscopy and theory, we determined the lithiation state of a $\mathrm{LiFePO}_{4}$ cathode and surrounding $0.5 \mathrm{M} \mathrm{Li}_{2} \mathrm{SO}$ aqueous electrolyte in real time with nanoscale resolution during electrochemical charge and discharge (Figure 3). We follow lithium transfer between electrode and electrolyte and image charging dynamics in the cathode. We observe competing delithiation mechanisms such as core-shell and anisotropic growth occurring in parallel for different particles under the same conditions. This technique represents a general approach for the operando 
nanoscale imaging of electrochemically active ions in the electrode and electrolyte in a wide range of electrical energy storage systems. [4]

\section{References:}

[1] N. de Jonge and F. Ross, Nature Nanotechnology 6 (2011) 695.

[2] C.M. Wang, et al. Journal of Materials Research 25 (2010) 1541.

[3] M.E. Holtz, et al. Microscopy and Microanalysis 19 (2013) 1027.

[4] Work supported by the Energy Materials Center at Cornell, DOE EFRC BES (DE-SC0001086). EM

Facility support from the NSF MRSEC program (DMR 1120296).
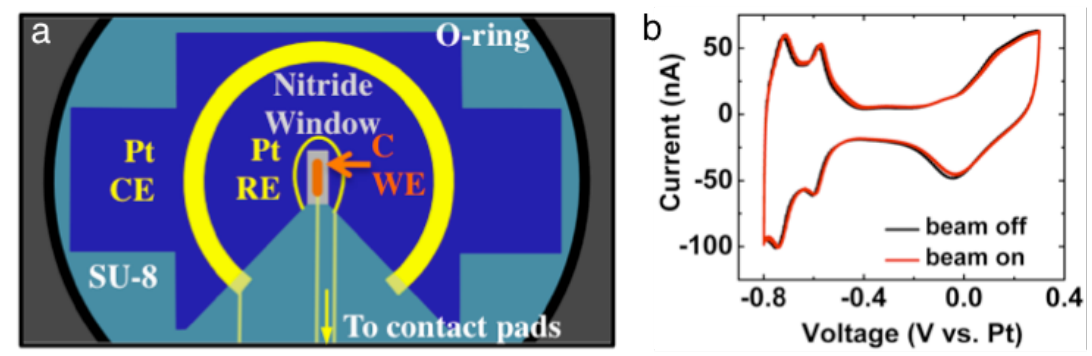

Figure 1. Electrochemical chip design and in situ electrochemistry data. (a) Schematic of the top chip with three patterned electrodes optimized for electrochemistry. (b) Cyclic voltammetry of platinum in sulfuric acid, with the expected hydrogen adsorption and desorption peaks reproduced.
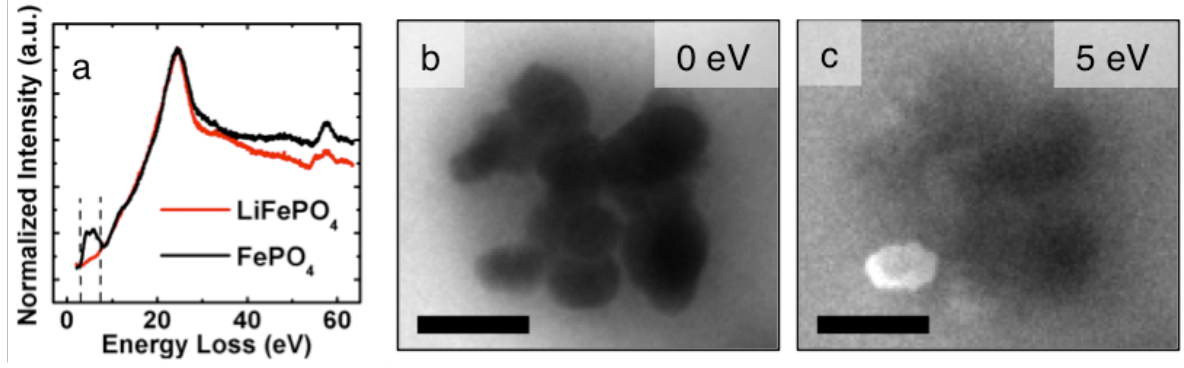

Figure 2. Spectroscopy of $\mathrm{LiFePO}_{4}$ and $\mathrm{FePO}_{4}$. (a) Ex situ EELS show a $5 \mathrm{eV}$ peak for $\mathrm{FePO}_{4}$ but not $\mathrm{LiFePO}_{4}$. EFTEM through liquid with a $5 \mathrm{eV}$ energy slit around (b) $0 \mathrm{eV}$ where the particles seem homogeneous and (c) $5 \mathrm{eV}$, which highlights the delithiated regions of $\mathrm{FePO}_{4}$. Scale bar is $200 \mathrm{~nm}$.

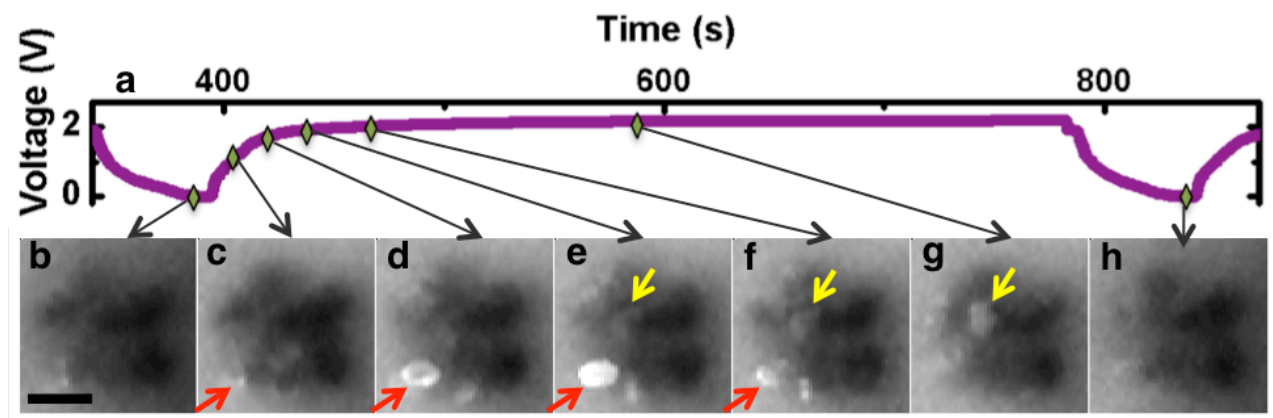

Figure 3. Observation of the evolution of a cluster of $\mathrm{LiFePO}_{4} / \mathrm{FePO}_{4}$ during one charge/discharge cycle. The voltage profile measured is shown in (a). (b-h) show the 5 eV EFTEM images with a $200 \mathrm{~nm}$ scale bar, where (b) is completely discharged. The red arrows in (c-f) correspond to core-shell type structures, and the yellow arrows in (e-f) show delithiation propagating in one direction through the particle. In the discharged state, the delithiated electrolyte appears dark as lithium is removed from solution as it intercalates the cathode material. 